\title{
Application of Bifurcation Theory to the High-Angle-of-Attack Dynamics of the F-14
}

\author{
C. C. Jahnke* \\ Clarkson University, Potsdam, New York 13699 \\ and \\ F. E. C. Culick $\dagger$ \\ California Institute of Technology, Pasadena, California 91125
}

\begin{abstract}
Bifurcation theory has been used to study the nonlinear dynamies of the F-14. An 8 degree-of-freedom model that does not include the control system present in operational F-14's has been analyzed. The aerodynamic model, supplied by NASA, includes nonlinearities as functions of the angles of attack and sideslip, the rotation rate about the velocity vector, and the elevator deflection. A continuation method has been used to calculate the steady states of the F-14 as continuous functions of the elevator deflection. Bifurcations of these steady states have been used to predict the onset of wing rock, spiral divergence, and jump phenomena that cause the aircraft to enter a spin. A simple feedback control system was designed to eliminate the wing rock and spiral divergence instabilities. The predictions were verified with numerical simulations.
\end{abstract}

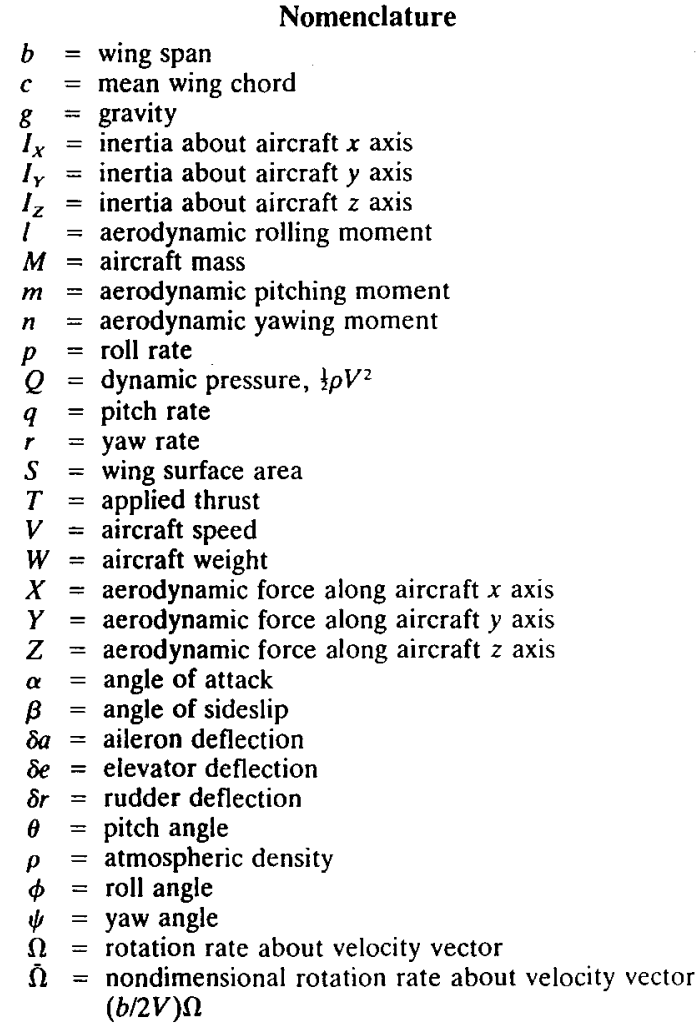

Received Feb. 12, 1992; revision received Oct. 6, 1992; accepted for publication Oct. 7, 1992. Coypright (C) 1992 by the American Institute of Aeronautics and Astronautics, Inc. All rights reserved.

*Assistant Professor, Mechanical and Aeronautical Engineering. Member AIAA.

tProfessor, Jet Propulsion and Mechanical Engineering. Fellow AIAA.

\section{Introduction}

$\mathbf{N}$ ONLINEAR dynamics are central to several important aircraft motions, including roll-coupling and stall/spin phenomena. Inertial coupling is the most important nonlinearity in roll-coupling instabilities, while stall/spin phenomena involve both nonlinear aerodynamics and inertial coupling. Linearized equations of motion cannot be used to analyze these phenomena. Indeed, roll-coupling instabilities were first discovered in flight, often with fatal results, because the linearized equations of motion used for analysis at that time did not contain the instability.

Phillips ${ }^{1}$ first analyzed the roll-coupling problem by treating the roll rate as a parameter in the linearized pitching and yawing moment equations. His analysis showed that aircraft with low inertia in roll could experience instabilities in pitch or yaw for certain critical roll rates. Inertial coupling also results in large sideslip deviations which cause high loads on the vertical tail.

Much of the subsequent research was devoted to predicting the maximum tail loads during maneuvers involving roll coupling. The typical method of analysis was to run many numerical simulations using simplified equations of motion that retained only nonlinearities important to roll-coupling phenomena. Gates and Minka ${ }^{2}$ calculated the steady states of simplified equations of motion for an aircraft and showed that the jump phenomena associated with roll-coupling instabilities resulted in the aircraft jumping from one steady state to another.

Subsequent researchers expanded the techniques of Gates and Minka ${ }^{2}$ to analyze more complete aerodynamic models. Young et al. ${ }^{3}$ developed an iterative technique for determining the steady states of a simplified set of equations of motion. The simplifications involved neglecting the effects of gravity and assuming a constant aircraft speed. Young et al. ${ }^{3}$ analyzed an aerodynamic model that only included nonlinearities as a function of the angle of attack. Their results allowed them to predict both the control surface deflections at which an instability would occur and the final state of the aircraft after the instability. The global nature of the results provided a qualitative understanding of the dynamics of the aircraft and clearly showed the benefits of calculating the steady states of an aircraft.

Analysis of the staly/spin phenomena developed along the same general pattern as analysis of the roll-coupling instabil- 
ity. Initially, most work involved numerical simulations of spin entry and attempts to determine recovery techniques. Early simulations of spin entry and recovery compared poorly with spin tunnel and flight tests. The reason for the disparities between the numerical simulations and flight tests was determined by Chambers et al. ${ }^{4}$ who showed that rotary balance data need to be included in the aerodynamic model in order to correctly model the aerodynamics during a spin. In particular, the nonlinearity of the yawing moment as a function of the yaw rate needs to be included in the aerodynamic model.

Recent analyses of stall/spin phenomena have involved attempts to determine the steady spin modes of aircraft. Adams ${ }^{5}$ developed an iterative search technique for determining the steady-state spins of several aircraft. His results compared poorly with flight tests because he did not include rotary balance data in his aerodynamic models. Tischler and Barlow ${ }^{6}$ developed a graphical technique for determining the steady spin modes of several general aviation aircraft. Rotary balance data were included in the aerodynamic models and the results compared well with flight test results.

A major shortcoming of the above techniques is that they all require some type of simplification of the equations of motion and/or the aerodynamic models. Continuation methods are numerical techniques for calculating the steady states of systems of ordinary differential equations and have recently been used to study roll-coupling instabilities and high-angleof-attack instabilities. Carroll and Mehra ${ }^{7}$ were the first to use a continuation technique to calculate the steady states of an aircraft. They determined the steady states of a variable sweep fighter and the F-4. By studying the steady states of these two aircraft they were able to show that wing rock occurs near the stall angle of attack due to a Hopf bifurcation of the trim steady state. Carroll and Mehra ${ }^{7}$ also calculated the steady spin modes for the aircraft and were able to predict both the control surface deflections at which the aircraft would undergo stall/spin divergence and the resulting steady state of the aircraft. They then developed recovery techniques using their knowledge of the steady spin modes for the aircraft. Control surface interconnects or control surface limits could also be designed based on the knowledge of the control surface deflections which resulted in stall/spin divergence.

Guicheteau ${ }^{8,9}$ has used continuation methods and bifurcation theory in studying the nonlinear dynamics of a realistic aircraft model that includes unsteady aerodynamic coefficients. The effects of a lateral offset of the c.g. and the effects of engine torque on spin entry and recovery have been studied. ONERA is using a German-French Alpha-Jet combat aircraft to corroborate the results obtained with the continuation method. Planeaux ${ }^{10.11}$ has used bifurcation analysis to study the periodic motions of model fighter aircraft. This is particularly relevant to understanding the spin behavior of an aircraft, as most aircraft have periodic spin modes. Planeaux has also analyzed aircraft models that contained control aug. mentation systems. The model used in the calculations was a simplified version of the control augmentation system in an actual aircraft, but the inclusion of a control system in the aircraft model was a major step towards realistic aircraft models. It is interesting to note that the qualitative nature of the steady states was not changed by the control augmentation system. The quantitative nature of the results could be very different depending on whether or not a control system is included in the model and the stability of a given steady state can be changed by the control system. Planeaux was able to change the control surface deflections at which wing rock occurred by providing roll rate feedback to the aileron or differential tail.

In this work we use a continuation method to determine the steady states of the F-14 as functions of the elevator deflection and bifurcations of these steady states. Results from dynamical systems theory are used to predict the nature of the instabilities caused by the bifurcations and the response of the aircraft after a bifurcation is encountered. Numerical simulations are used to verify the predictions. Instabilities during longitudinal maneuvers were shown to cause wing rock and spiral divergence. Steady spins were determined as functions of the elevator deflection, but attempts to recover from developed spins proved unsuccessful.

\section{Theoretical Background}

\section{Dynamical Systems Theory}

Dynamical systems theory provides a methodology for studying systems of ordinary differential equations. Many systems have been studied using the techniques of dynamical systems theory, but the techniques have not been widely used to study the equations of motion for an aircraft. The important ideas of dynamical systems theory used in this report will be introduced in the following paragraphs. More information on dynamical systems theory can be found in the book of Guckenheimer and Holmes. ${ }^{12}$

The first step in analyzing a system of nonlinear differential equations, in the dynamical systems theory approach, is to calculate the steady states of the system and their stability. Steady states of a system can be found by setting all time derivatives equal to zero and solving the resulting set of algebraic equations. The Hartman-Grobman theorem (Guckenheimer and Holmes, ${ }^{12}$ Chap. 1, p. 13) proves that the local stability of a steady state can be determined by linearizing the equations of motion about the steady state and calculating the eigenvalues. A steady state is locally stable if the real parts of all the eigenvalues of the linearized system are negative. If the real part of any eigenvalue of the linearized system is positive, the steady state is locally unstable. In the neighborhood of a steady state the system will be attracted to the steady state if the steady state is stable and repelled from the steady state if the steady state is unstable.

The implicit function theorem (Ioos and Joseph, ${ }^{13}$ Chap. 2 , pp. 13,14) proves that the steady states of a system are continuous functions of the parameters of the system at all steady states where the linearized system is nonsingular. A singular linearized system is characterized by a zero eigenvalue. Thus, the steady states of the equations of motion for an aircraft are continuous functions of the control surface deflections. Stability changes can occur as the parameters of the system are varied in such a way that the real parts of one or more eigenvalues of the linearized system change sign. Changes in the stability of a steady state lead to qualitatively different responses for the system and are called bifurcations. Stability boundaries can be determined by searching for steady states which have one or more eigenvalues with zero real parts.

There are many types of bifurcations and each has a different effect on the response of the system. Qualitative changes in the response of the system can be predicted by determining how many and what types of eigenvalues have zero real parts at the bifurcation point. Bifurcations for which one real eigenvalue is zero lead to the creation or destruction of two or more steady states. Bifurcations for which one pair of complex eigenvalues has zero real parts can lead to the creation or destruction of periodic motions.

Bifurcations for which more than one real eigenvalue or more than one pair of complex eigenvalues have zero real parts lead to very complicated behavior and are beyond the scope of this report. Three types of bifurcations were found to occur in the steady states of the F-14: 1) saddle-node, 2) pitchfork, and 3) Hopf. Guckenheimer and Holmes ${ }^{12}$ provide a thorough introduction to the various types of bifurcations that occur in nonlinear systems and their effects on the dynamics of the system.

\section{Continuation Methods}

Continuation methods are a direct result of the implicit function theorem, which proves that the steady states of a system are continuous functions of the parameters of the sys- 


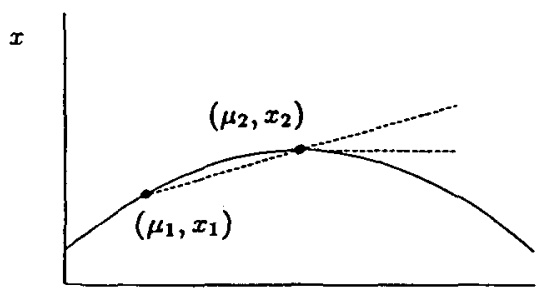

Fig. 1 Graphical representation of continuation method.

tem at all steady states except for steady states at which the linearized system is singular. The general technique is to fix all parameters but one and trace the steady states of the system as a function of this parameter. If one steady state of the system is known, a new steady state can be approximated by linear extrapolation from the known steady state (see Fig. 1). The slope of the curve at the steady state can be determined by taking the derivative of the equation given by setting all time derivatives equal to zero.

If two steady states are known, a new steady state can be approximated by linear extrapolation through the two known steady states as shown in Fig. 1. This technique is much more efficient than calculating the exact slope of the curve at the known steady state, which requires a matrix inversion. Errors between the approximate steady state and the true steady state can be reduced with Newton's method. The stability of each steady state can be determined by calculating the eigenvalues of the linearized system. Any changes in stability from one steady state to the next will signify a bifurcation. There are several continuation method algorithms, in this work we use the algorithm developed by Doedel and Kerneve $z^{14}$ which is based on the work of Keller. ${ }^{15}$

\section{Model of Aircraft Dynamics}

The purpose of this work has been to use bifurcation theory to analyze the equations of motions for an aircraft. This work concentrated on the high-angle-of-attack dynamics of the F14 for several reasons. The main reason is that high-angle-ofattack dynamics are inherently nonlinear and cannot be analyzed by the traditional linear techniques. Also, with the recent emphasis on developing jet fighters that can maneuver at high angles of attack it was felt that results on high-angleof-attack flight would be particularly relevant.

The equations of motion used in this study assumed a rigid aircraft, no applied thrust, and constant atmospheric density. The equations were written in a principal axis system and consist of

rotational equations

$$
\begin{gathered}
\dot{p}=\frac{I_{Y}-I_{Z}}{I_{X}} q r+\frac{l}{I_{X}} \\
\dot{q}=\frac{I_{Z}-I_{X}}{I_{Y}} p r+\frac{m}{I_{Y}} \\
\dot{r}=\frac{I_{X}-I_{Y}}{I_{Z}} p q+\frac{n}{I_{Z}}
\end{gathered}
$$

translational equations

$$
\begin{aligned}
\dot{\alpha} & =q-(p \cos \alpha+r \sin \alpha) \tan \beta \\
& +\frac{1}{M V \cos \beta}(Z \cos \alpha-X \sin \alpha) \\
& +\frac{g}{V \cos \beta}(\sin \alpha \sin \theta+\cos \alpha \cos \theta \cos \phi)
\end{aligned}
$$

$$
\begin{aligned}
\dot{\beta} & =p \sin \alpha-r \cos \alpha+\frac{1}{M V}[Y \cos \beta \\
& -(X \cos \alpha+Z \sin \alpha) \sin \beta] \\
& +\frac{g}{V}(\cos \alpha \sin \beta \sin \theta+\cos \beta \cos \theta \sin \phi \\
& -\sin \alpha \sin \beta \cos \theta \cos \phi) \\
\dot{V} & =\frac{1}{M}[(X \cos \alpha+Z \sin \alpha) \cos \beta+Y \sin \beta] \\
& +g(\sin \beta \cos \theta \sin \phi-\cos \alpha \cos \beta \sin \theta \\
& +\sin \alpha \cos \beta \cos \theta \cos \phi)
\end{aligned}
$$

Euler angles

$$
\begin{aligned}
\dot{\theta} & =q \cos \phi-r \sin \phi \\
\phi & =p+(q \sin \phi+r \cos \phi) \tan \theta \\
\psi & =(q \sin \phi+r \cos \phi) \sec \theta
\end{aligned}
$$

The equation for the time rate of change of $\psi$ is decoupled from the other equations, so the system can be reduced fror: a ninth-order system to an eighth-order system. The steady states presented in this article are steady states of the first eight equations, while the yaw angle will, in general, vary with time.

The aerodynamic model used in this work was supplied by NASA Ames Dryden Flight Research Center and is the aerodynamic model used in their flight simulators. The model includes nonlinearities as functions of the angles of attack and sideslip, the rotation rate about the velocity vector, and the elevator deflection. Data were reported for angles of attack from $0-90 \mathrm{deg}$, angles of sideslip from -20 to $+20 \mathrm{deg}, \bar{\Omega}$, from -0.54 to +0.54 , and elevator deflections from -30 to +10 deg. Mach number effects were included in the aerodynamic model provided by NASA, but were not included in this work. This limited the Mach number of the results presented here to be less than 0.60 .

Three aerodynamic data bases are included in the model: 1) low angle of attack, 2) high angle of attack, and 3) rotary balance data. The low-angle-of-attack aerodynamic mode! is reported for angles of attack from 0 to $55 \mathrm{deg}$, angles of sideslip from -20 to $+20 \mathrm{deg}$, and elevator deflections from -30 to $+10 \mathrm{deg}$ and has the form

$$
\begin{aligned}
& X=X(\alpha, \beta)+q X_{q}(\alpha) \\
& +\left[\begin{array}{cc}
X_{\delta e .1}(\alpha, \beta) \delta e ; & \delta e \geq-10 \\
-10 X_{\delta e, 1}(\alpha, \beta)+(\delta e+10) X_{\delta e, 2}(\alpha, \beta) ; & \delta e \leq-10
\end{array}\right] \\
& Z=Z(\alpha, \beta)+\bar{q} Z_{q}(\alpha) \\
& +\left[\begin{array}{cc}
\delta e Z_{\delta e, 1}(\alpha, \beta) ; & \delta e \geq-10 \\
-10 Z_{\delta e, 1}(\alpha, \beta)+(\delta e+10) Z_{\delta e, 2}(\alpha, \beta) ; & \delta e \leq-10
\end{array}\right] \\
& m=m(\alpha, \beta)+\tilde{q} m_{q}(\alpha) \\
& +\left[\begin{array}{cc}
\delta e m_{\delta e, 1}(\alpha, \beta) ; & \delta e \geq-15 \\
-15 m_{\delta e, 1}(\alpha, \beta)+(\delta e+15) m_{\delta e, 2}(\alpha, \beta) ; & \delta e \leq-15
\end{array}\right] \\
& Y=Y(\alpha, \beta)-\delta a Y_{\delta a}(\alpha, \beta)+\delta r Y_{\delta r}(\alpha, \beta) \\
& +\bar{r} Y_{r}(\alpha)+\bar{p} Y_{p}(\alpha) \\
& l=l(\alpha, \beta)+\beta D l_{\beta, 2}(\alpha)-\delta a\left[l_{\delta a, 1}(\alpha, \beta)+l_{\delta a, 2}(\alpha, \delta e)\right] \\
& +\delta r l_{\delta r}(\alpha, \beta)+\bar{r} l_{r}(\alpha)+\bar{p} l_{p}(\alpha) \\
& n=n(\alpha, \beta, \delta e)-\delta a\left[n_{\delta a, 1}(\alpha, \beta)+n_{\delta a, 2}(\alpha, \delta e)\right] \\
& +\delta r n_{\delta r}(\alpha, \beta, \delta e)+\bar{r} n_{r}(\alpha)+\bar{p} n_{p}(\alpha)
\end{aligned}
$$


where

$$
\begin{aligned}
\bar{q} & =q-\Omega \sin \beta \\
\bar{r} & =r-\Omega \sin \alpha \cos \beta \\
\bar{p} & =p-\Omega \cos \alpha \cos \beta \\
\Omega & =(p \cos \alpha+r \sin \alpha) \cos \beta+q \sin \beta
\end{aligned}
$$

The variables $\bar{q}, \bar{r}$, and $\vec{p}$ are used in the forced oscillation aerodynamic terms to keep the rotary balance aerodynamic model and the forced oscillation aerodynamic model independent of each other.

The high-angle-of-attack aerodynamic model is reported for angles of attack from 55 to $90 \mathrm{deg}$, angles of sideslip from -20 to $+20 \mathrm{deg}$, and elevator deflections from 0 to $-30 \mathrm{deg}$ and has the form

$$
\begin{aligned}
X & =X(\alpha, \beta)+\delta e X_{\delta e}(\alpha, \beta) \\
Z & =Z(\alpha, \beta)+\bar{q} Z_{q}(\alpha)+\delta e Z_{\delta e}(\alpha, \beta) \\
m & =m(\alpha, \beta)+\bar{q} m_{q}(\alpha)+\dot{\alpha} m_{\alpha}(\alpha)+\delta e m_{b e}(\alpha, \beta) \\
Y & =Y(\alpha, \beta)-\delta a Y_{\delta a}(\alpha, \beta)+\bar{r} Y_{r}(\alpha)+\bar{p} Y_{p}(\alpha) \\
l & =l(\alpha, \beta)-\delta a l_{\delta a}(\alpha, \beta)+\bar{r} l_{r}(\alpha)+\bar{p} l_{p}(\alpha) \\
n & =n(\alpha, \beta, \delta e)-\delta a n_{\delta a}(\alpha, \beta)+\bar{r} n_{r}(\alpha)+\bar{p} n_{p}(\alpha)
\end{aligned}
$$

Rotary balance data is reported for angles of attack from 0 to $90 \mathrm{deg}$, angles of sideslip from -20 to $+20 \mathrm{deg}$, elevator deflections from -30 to $+10 \mathrm{deg}$, and nondimensional roll rates about the velocity vector from -0.54 to +0.54 , where the nondimensional rotation rate is defined as

$$
\bar{\Omega}=(b / 2 V) \Omega
$$

The rotary balance aerodynamic model has the form

$$
\begin{aligned}
X & =0.0 \\
Z & =-\operatorname{DCNORM}(\alpha, \bar{\Omega}) \\
m & =\operatorname{DCMR}(\alpha, \bar{\Omega})+\operatorname{DCMRB}[\alpha, \bar{\Omega} \operatorname{sign}(\beta)]|\beta| \\
Y & =\operatorname{DCYR}(\alpha, \bar{\Omega})+\operatorname{DCYRIS}(\alpha, \bar{\Omega}) \operatorname{AKCYIS}(\bar{\Omega}, \delta e) \\
l & =\operatorname{DCLR}(\alpha, \bar{\Omega})+\operatorname{DCLRIS}(\alpha, \bar{\Omega}) \operatorname{AKCL}(\delta e) \\
& +\operatorname{DCLRDD}[\alpha, \bar{\Omega} \operatorname{sign}(\delta a)]|\delta a / 7| \\
n & =\operatorname{DCNR}(\alpha, \bar{\Omega})+\operatorname{DCNRIS}(\alpha, \bar{\Omega}) \operatorname{AKCNIS}(\bar{\Omega}, \delta e) \\
& +\operatorname{AKCNB}(\alpha, \beta) \operatorname{DCNRB}(\alpha, \bar{\Omega}, \beta) \\
& +\operatorname{DCNRDD}[\alpha, \bar{\Omega} \operatorname{sign}(\delta a), \delta e]|\delta a / 7|
\end{aligned}
$$

One important characteristic of this aerodynamic model is that the rudder is ineffective at angles of attack greater than $55 \mathrm{deg}$. This is a significant limitation to developing spin recovery techniques for the F-14. Jahnke ${ }^{16}$ contains more details on the aerodynamic model.

The aerodynamic coefficients must have a continuous first derivative for the continuation algorithm to converge, so the aerodynamic data were approximated with bicubic functions using an algorithm of Press et al. ${ }^{17}$ This type of fit can introduce large curvatures to the aerodynamic data. The data were approximated with linear interpolation in the time simulations to make sure the bifurcations predicted by the continuation method were not a result of the curvatures introduced by the data fit.

\section{Results}

The dynamics of the F-14 have been studied by determining the steady states of the equations of motion and seeking bifurcations. The steady states are plotted as functions of the

aileron or elevator deflection. Atmospheric density is held constant at $0.53 \mathrm{~kg} / \mathrm{m}^{3}$, which corresponds to an altitude of $20,000 \mathrm{ft}$, and the applied thrust is zero for the results presented here. Including nonzero applied thrust in the analysis would change the steady-state velocity of the aircraft, but not the qualitative nature of the results. Jahnke ${ }^{18}$ has shown that for subsonic speeds, both high-angle-of-attack and roll-coupling instabilities are insensitive to airspeed. Also, since the aerodynamic model used in this study was limited to Mach numbers below 0.60 , a wider range of elevator deflections could be analyzed when zero applied thrust was used in the analysis. The small Canards included in operational F-14s to provide longitudinal stability are not included in the aircraft model used in this study. Also, the spoilers are retracted and the wings fully swept forward for the results presented here.

\section{Longitudinal Maneuvers}

Figure 2 shows the steady states of the F-14 that are at low angles of attack. Steady states represented by curve 1 are the longitudinal trim conditions and the steady states represented by curves $2 N$ and $2 P$ represent spirally divergent motions. The $N$ and $P$ are used to denote steady states with negative and positive roll rates, respectively. Figure 2 shows that for elevator deflections greater than $7 \mathrm{deg}$ the trim conditions of the F-14 are stable. The steady states represented by curve 1 were calculated up to an elevator deflection of $-40 \mathrm{deg}$. A smaller range is shown in Fig. 2 so that the instabilities that occur for small elevator deflections can be clearly seen. The trim condition for a given elevator deflection can be determined by drawing a vertical line representing the desired elevator deflection on each plot; each intersection of this line with the curve of steady states gives a possible steady state of the aircraft.

For elevator deflections between -6.7 and $-5.4 \mathrm{deg}$, the steady-state trim conditions of the F-14 are unstable as a result of two Hopf bifurcations. Hopf bifurcations can lead to periodic motions, so it is possible that for elevator deflections between -6.7 and -5.4 deg the F-14 will undergo periodic
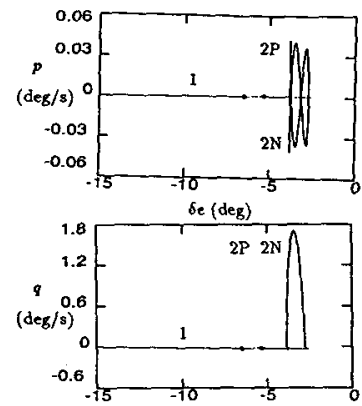

$\delta e(\mathrm{deg})$
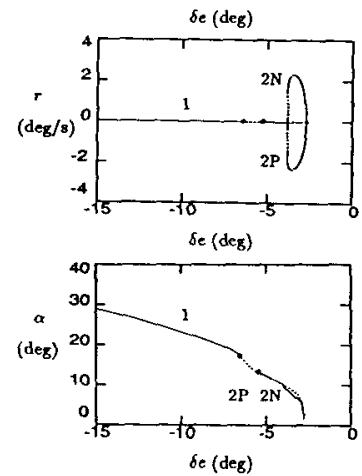

Fig. 2 Steady states which are at low angles of attack, $\delta a=0, \delta r$ = 0; - stable; --- unstable; • Hopf bifurcation.
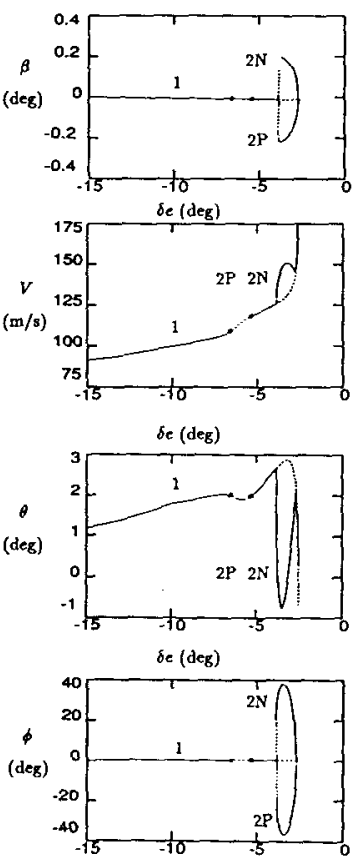

$\delta e$ (deg) 

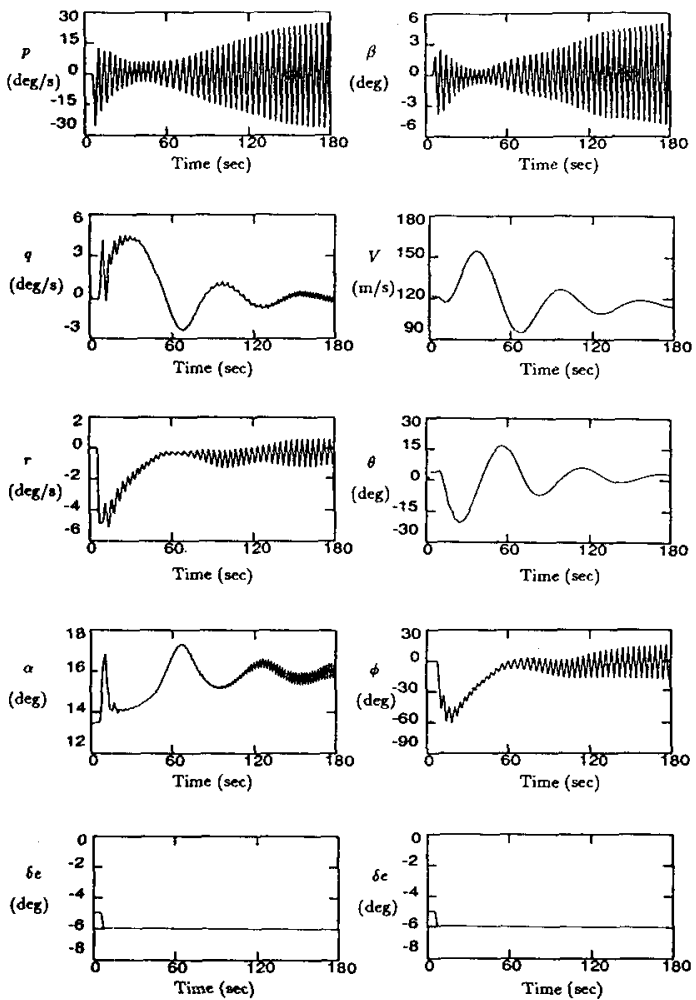

Fig. 3 Simulation of wing rock instability, $\delta r=0$.

motions. Figure 3 shows a time simulation in which the elevator deflection is changed from -5 to $-6 \mathrm{deg}$, putting the aircraft in a region of unstable steady states. The figure shows that a slowly developing wing rock is present for an elevator deflection of $-6 \mathrm{deg}$. The oscillations grow slowly and have a period of about $4 \mathrm{~s}$, so they would not be a danger to pilots. Note that the magnitude and frequency of these oscillations could change if the airspeed was increased (by applying thrust) or if the atmospheric density was changed (due to changing the altitude).

For elevator deflections between -3 and -4 deg there are three possible steady states for the aircraft. The trim conditions (curve 1) are unstable while the two steady states representing spiral divergence are stable. Thus, for elevator deflections between -3 and -4 deg the F-14 would experience spiral divergence. A time simulation of this is shown in Fig. 4. The roll angle of the aircraft changes rapidly in response to a one-tenth of a degree aileron perturbation and then continues to slowly increase. The pitch angle and velocity also change as the aircraft enters a shallow spiral. This instability grows very slowly and could be controlled by a pilot.

The wing rock and spiral divergence instabilities could also be controlled with a simple feedback control system. Wing rock may be a result of low damping in roll, ${ }^{19}$ while spiral divergence may be a result of insufficient dihedral effect. ${ }^{20}$ Feedback to the ailerons can be used to supplement both of these stability derivatives. Roll rate feedback can be used to increase the effective roll damping and sideslip feedback can be used to supplement the dihedral effect. Figure 5 shows the steady states that are at low angles of attack when sideslip and roll rate feedback to the ailerons are included in the aircraft model. The figure shows that both the wing rock instability and the spiral divergence instability have been eliminated through the use of feedback to the ailerons. It should be noted that the effect of the control system was computed
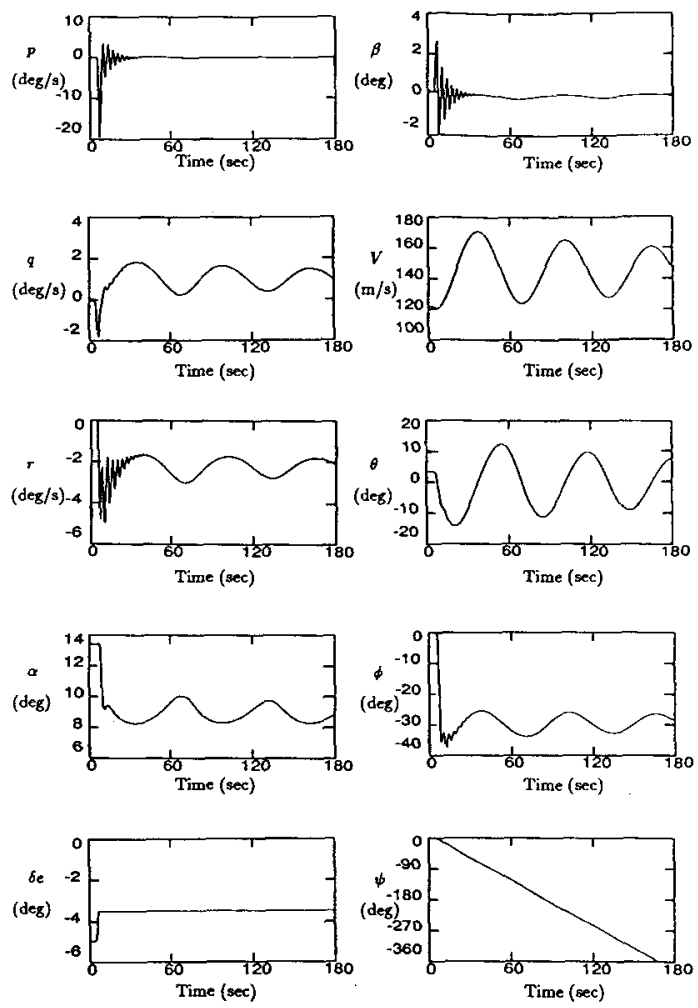

Fig. 4 Simulation of spiral divergence instability, $\delta r=0$.
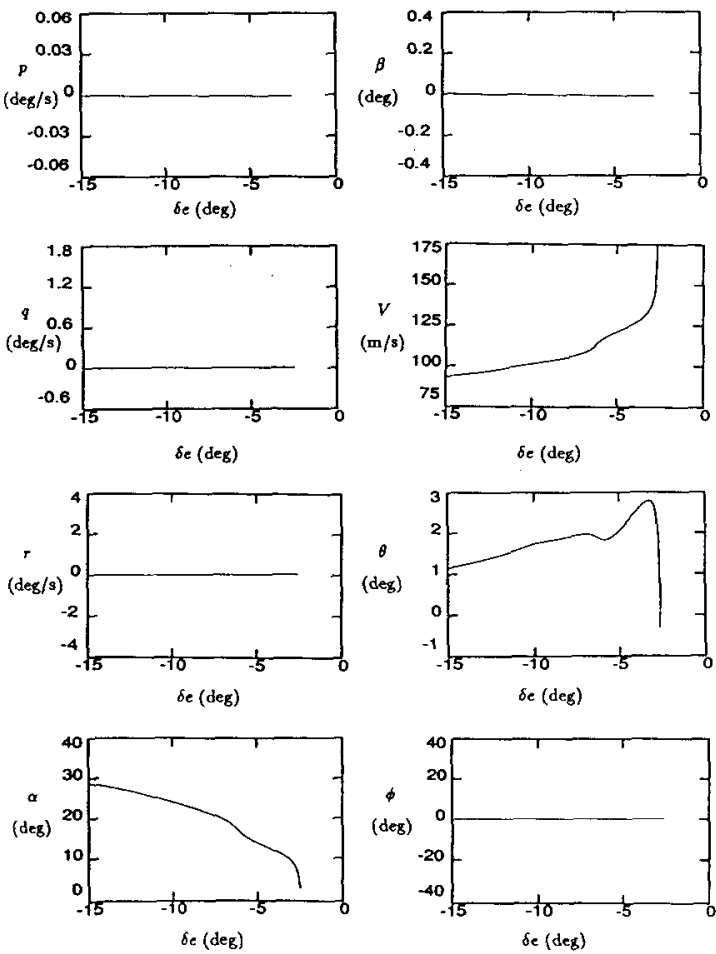

Fig. 5 Steady states with sideslip and roll rate feedback to the ailerons, $\delta r=0, \delta a=-0.1 p-0.3 \beta ;-$ stable. 
on the steady states of the nonlinear equations of motion. Continuation methods make it possible to determine the effects of a control system on nonlinear systems as opposed to the classical method of determining the effects of a control system on the linearized equations of motion.

\section{Lateral Maneuvers}

Figure 6 shows the steady states of the F-14 as a function of aileron deflection for an elevator deflection of $-10 \mathrm{deg}$ and zero rudder deflection. The figure shows that multiple steady states exist for most aileron deflections. For example, a vertical line representing $0 \mathrm{deg}$ of aileron deflection intersects five steady states. Three of these steady states are stable so the aircraft could exhibit either of these three steady states for 0 aileron deflection. Note that five steady states also exist in Fig. 2 for a vertical line representing an elevator deflection of $-10 \mathrm{deg}$, but only one curve of steady states is shown in the figure.

One stable steady state at 0 aileron deflection represents the trim condition for an elevator deflection of -10 deg (i.e., $p=q=r=\beta=\phi=0$ ). The other two stable steady states represent spins. This can be seen by noting that these steady states all have angles of attack near $80 \mathrm{deg}$ and large steadystate yaw rates. The segment of stable steady states containing the trim condition for an elevator deflection of $-10 \mathrm{deg}$ only exists for aileron deflections between -12 and +12 deg because of two saddle-node bifurcations that occur at aileron deflections of -12 and +12 deg.

For example, see the steady-state angles of attack shown in Fig. 6. If the aircraft is trimmed at an elevator deflection of -10 deg the steady-state angle of attack will be given by the angle of attack at 0 aileron deflection contained in the curve of low-angle-of-attack steady states. If the aileron deflection is increased slowly enough, the steady state of the aircraft will be given by the curve of stable low-angle-of-attack steady states up to an aileron deflection of $12 \mathrm{deg}$. For aileron deflections greater than $12 \mathrm{deg}$, the steady states that are at low angles of attack do not exist, so the aircraft will jump to
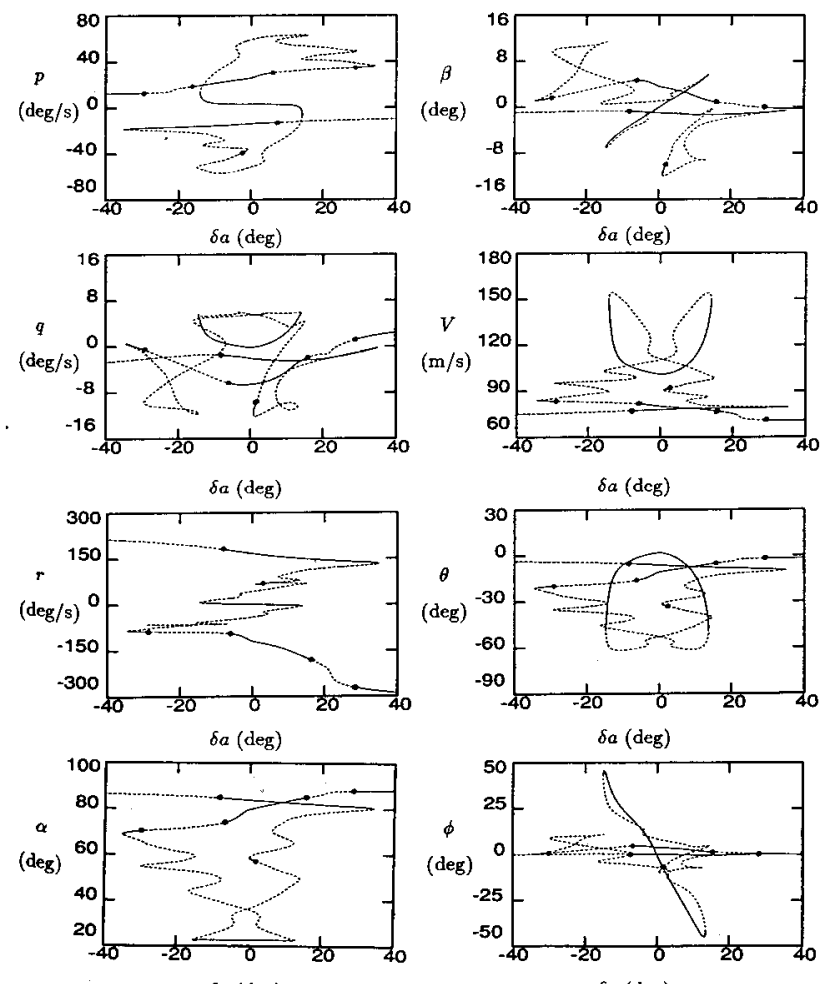

$\delta a(\mathrm{deg})$

$\delta a(\operatorname{deg})$

Fig. 6 Steady states for lateral maneuvers with $\delta e=-10, \delta r=0$; - stable; -.. unstable; $\bullet$ Hopf bifurcation.
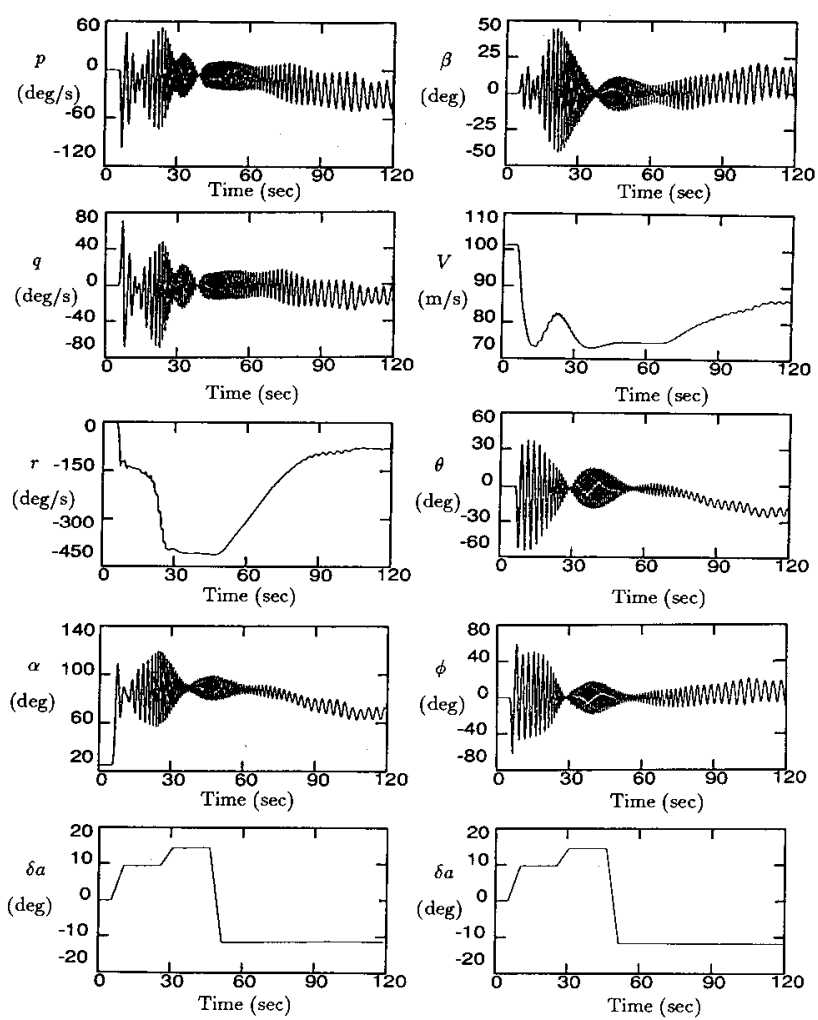

Fig. 7 Simulation of instability during a lateral maneuver with $\delta e$ $=-10, \delta r=0$.

a new stable motion. This new motion could be either a stable steady state or some type of time-dependent motion.

Figure 7 shows a simulation of the maneuver described above. The simulation shows that as the aileron deflection is increased to $10 \mathrm{deg}$, the aircraft enters a spin, while the steadystate analysis predicted spin entry at an aileron deflection of $12 \mathrm{deg}$. The difference between the critical aileron deflection predicted by the steady-state analysis and the critical aileron deflection predicted by the time simulation could either be a result of the transient aileron deflection in the simulation or a result of the different aerodynamic curve fits used in the continuation method algorithm and the simulation program.

A recovery from the spin is attempted by reducing the aileron deflection to $-12 \mathrm{deg}$, at which point the steady spin becomes unstable because of a Hopf bifurcation (see Fig. 6). The recovery is not successful because a stable oscillatory spin develops. Recall that the rudder is ineffective at angles of attack greater than $55 \mathrm{deg}$, so only the elevator and ailerons are available to attempt a recovery from a spin. The lack of rudder authority at high angles of attack could make it impossible to recover from a developed spin.

Since it is difficult or impossible to recover from a developed spin in the F-14, it is clearly desirable to avoid entering a spin. The saddle-node bifurcations that occur at aileron deflections of +12 and -12 deg were responsible for the aircraft entering a spin. If the aileron deflections were limited to values less than those at which the saddle-node bifurcations occur, it might be possible to avoid entering a spin. Fig. 8 shows the loci of elevator and aileron deflections at which the saddle-node bifurcations responsible for spin entry occur. This diagram could be used to put limits on the aileron deflection. Inclusion of a control system could change this figure so the results for an operational F-14 may be different.

Rudder deflection is applied during most lateral maneuvers and would, in general, change the control surface deflections at which bifurcations occur. Figure 9 shows the steady states of the aircraft as a function of aileron deflection for an elevator deflection of $-10 \mathrm{deg}$ and a rudder deflection of $-2 \mathrm{deg}$. The elevator deflection is the same for Figs. 6 and 9, so 


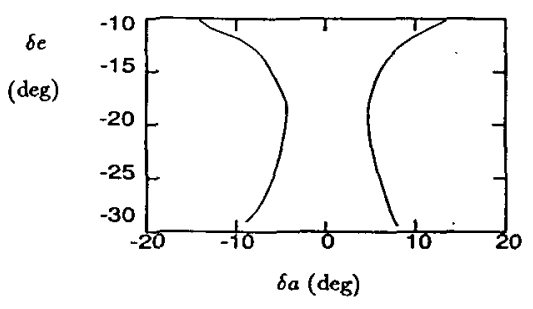

Fig. 8 Bifurcation loci for $\delta r=0$.
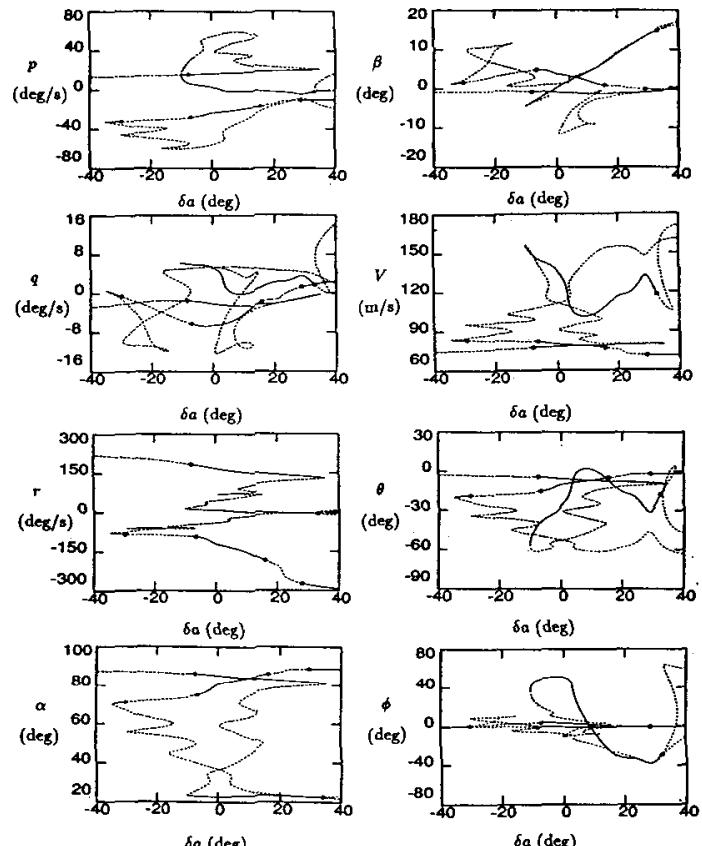

Fig. 9 Steady states for lateral maneuvers with $\delta e=-10, \delta r=$ -2 ; - stable; --- unstable; $\bullet$ Hopf bifurcation.

differences in the steady states in the two figures are a result of the different rudder deflections.

Applying 2 deg of negative rudder deflection has a dramatic effect on the steady states of the aircraft which are at low angles of attack. Figure 6 shows that with no rudder deflection, the steady states that are at low angles of attack only exist for aileron deflections between -12 and $+12 \mathrm{deg}$. When $2 \mathrm{deg}$ of negative rudder deflection are applied (see Fig. 9), steady states that are at low angles of attack exist for aileron deflections between -10 and $+30 \mathrm{deg}$. Thus, by using the rudder, it is possible to apply $18 \mathrm{deg}$ of extra positive aileron deflection. Note that steady states that have angles of attack greater than $55 \mathrm{deg}$ are the same for Figs. 6 and 9 because the rudder is ineffective for angles of attack greater than $55 \mathrm{deg}$.

Figure 10 shows the combination of rudder and aileron deflections that cause the steady states that are at low angles of attack to become unstable. For example, drawing a horizontal line representing 0 rudder deflection shows that saddlenode bifurcations cause the steady states that are at low angles of attack to become unstable for aileron deflections larger than +12 or -12 deg. Figure 10 could be used to put limits on the aileron deflection for a given rudder deflection, or it could be used to design an aileron-rudder interconnect such that the combinations of aileron and rudder deflections at which bifurcations occur could not be realized. Recall that
Fig. 10 is only valid for an elevator deflection of $-10 \mathrm{deg}$, but similar plots could be made for other elevator deflections.

\section{Steady Spin Modes}

Continuation methods require a known steady state as a starting point for the continuation procedure. It is usually easy to determine steady states that are at low angles of attack. Determining the steady spin modes for an aircraft is a more difficult task and it is usually not possible to be certain that all the steady spin modes of a particular aircraft have been determined.

The approach used to find the spin modes in this work was to guess an initial spin mode as a starting point for the continuation method algorithm, then let the algorithm run until either a true steady spin was determined or the algorithm ran into numerical problems. Spin modes determined by Adams $^{5}$ and Jahnke ${ }^{16,18}$ for other aircraft were used as a guide for picking the approximate spin modes.

Steady spin modes can also be obtained from Fig. 6. Recall that the steady states for 0 aileron deflection and angles of attack greater than $80 \mathrm{deg}$ are steady spin modes. It is also important to search for branches of steady spin modes that are not connected to the branch of steady states containing the trim conditions for the aircraft. For example, the curves

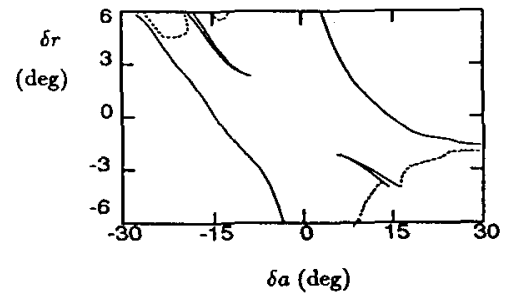

Fig. 10 Stability boundary for lateral maneuvers with $\delta e=-10$; saddle-node bifurcation; -.- Hopf bifurcation.
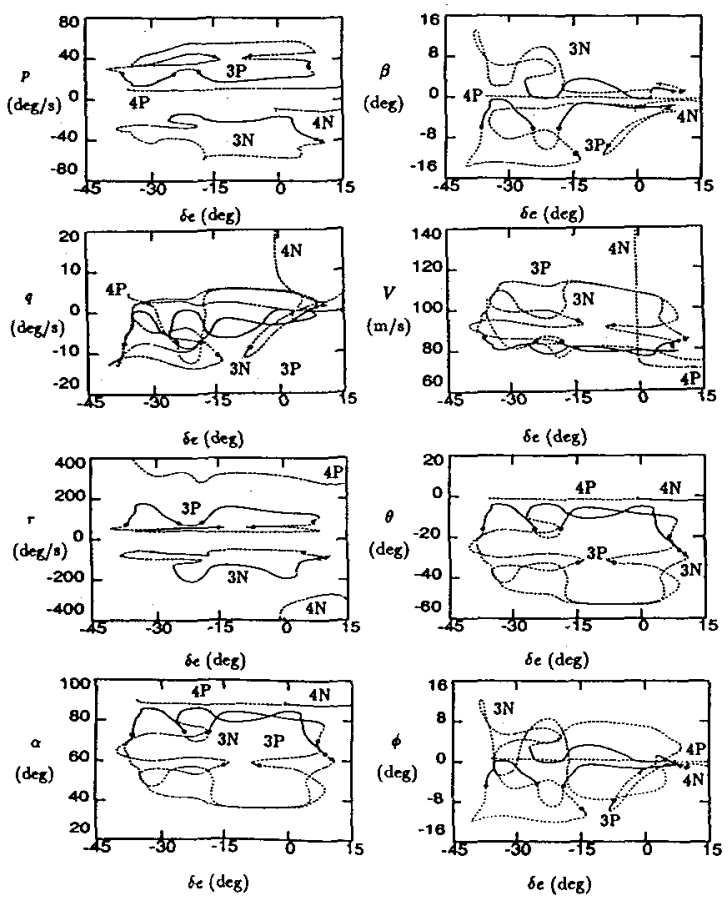

Fig. 11. Steady state spin modes for $\delta a=0, \delta r=0$; .- unstable; $\bullet$ Hopf bifurcation. 

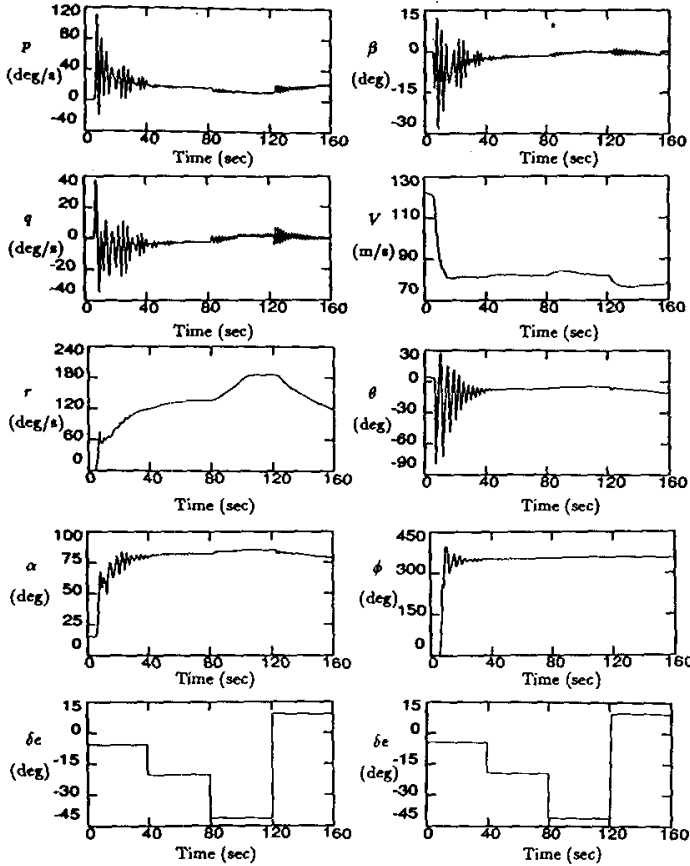

Fig. 12 Simulation of spin entry and attempted recovery with ailerons and rudder neutral.

$4 N$ and $4 P$ in Fig. 11 represent branches of steady spin modes that are not connected to branches $3 N$ and $3 P$. A major difficulty with nonlinear problems is that it is difficult if not impossible to be sure that all branches of steady states have been found.

Figure 11 shows the steady spin modes of the F-14 that were found in this work as a function of the elevator deflection. The asymmetry of the aerodynamic model is evident in the spin modes. If the aerodynamic model was symmetric, curves $3 N$ and $3 P$ and curves $4 N$ and $4 P$ would be symmetric. Spin modes represented by curves $4 N$ and $4 P$ are flat spins $(\alpha \approx$ $90 \mathrm{deg}$ ) at very high yaw rates. These spin modes were unstable for all control surface deflections used during the course of this work.

Spin modes given by curves $3 N$ and $3 P$ represent both flat spins $(\alpha \approx 90 \mathrm{deg})$ and steep spins $(\alpha \approx 50 \mathrm{deg})$, but only the flat spins are stable. Figure 11 shows that stable steady spins exist for almost the entire range of elevator deflections, so it may not be possible to recover from a developed spin with only elevator deflections for 0 aileron deflection. Recall that the rudder is ineffective for angles of attack greater than 55 deg.

Figure 12 shows an attempted spin recovery using only the elevator. A small perturbation in the aileron deflection for an elevator deflection of $-5 \mathrm{deg}$ causes the aircraft to enter a spin with a positive roll rate. The elevator deflection is first increased to $-20 \mathrm{deg}$ in an attempt to recover from the spin, because the steady spin modes with positive roll rates $(3 P)$ are unstable for an elevator deflection of $-20 \mathrm{deg}$ (see Fig. 11). This attempted recovery is not successful as the aircraft enters an oscillatory spin.

Increasing the elevator deflection to $-40 \mathrm{deg}$, for which no stable or unstable steady spin exists, is also unsuccessful in recovering from the spin because a stable oscillatory spin exists at this elevator deflection. Finally, full nose down elevator is applied ( $\delta e=-10 \mathrm{deg}$ ) but this is also unsuccessful in recovering from the spin. This example shows the need to determine the time-dependent spin modes along with the steady spin modes in order to develop spin recovery techniques.

\section{Conclusions}

The above results show the value of using continuation methods and dynamical systems theory for analyzing the equations of motion for an aircraft. The efficiency of the method makes it possible to analyze complicated aerodynamic models using the complete equations of motion for the entire range of control surface deflections. The results presented here were computed on a micro-VAX and it generally took about $1 \mathrm{~min}$ to compute curves of steady states such as those shown in Fig. 6. Simulations usually took 20 times as long, which shows the efficiency of calculating the steady states with continuation methods.

The method has great potential for designing control laws. Figures like Fig. 8 could be used to put limits on the control surface deflections so pilots stay away from jump phenomena. Simple feedback control systems can also be included in the aircraft model to determine the effects of control systems on the various instabilities. This could be particularly useful for designing control systems for high-angle-of-attack flight where the equations of motion are inherently nonlinear and results from traditional linear control theory might not be valid.

A knowledge of the control surface deflections that cause bifurcations can also be used to escape from motions caused by a jump in the state of the aircraft. No successful spin recovery technique was determined for this aircraft because of the presence of stable periodic spin modes. This points to the need to determine the control surface deflections that lead to the existence of stable periodic spins. Continuation methods can be extended to determine periodic motions as functions of the parameters of the system just as the fixed points have been found in this work.

\section{Acknowledgments}

This work was partly supported by CALTECH funds and partly supported by Grant NCC2-429 from NASA Ames Dryden Flight Research Center. The F-14 aerodynamic model was supplied by Joe Gera, Acting Assistant Branch Chief, Vehicle Technical Branch, NASA Ames Dryden Flight Research Center.

\section{References}

'Phillips, W. H., "Effect of Steady Rolling on Longitudinal and Directional Stability," NASA TN 1627, June 1948.

${ }^{2}$ Gates, O. B., and Minka, K., "Note on a Criterion for Severity of Roll-Induced Instability," Journal of the AerolSpace Sciences, May 1959 , pp. $287-290$

${ }^{3}$ Young, J. W., Schy, A. A., and Johnson, K. G., "PseudosteadyState Analysis of Nonlinear Aircraft Maneuvers," NASA TP 1758 Dec. 1980.

${ }^{4}$ Chambers, J. R., Bowman, J. S., and Anglin, E. L., "Analysis of the Flat Spin Characteristics of a Twin-Jet Swept-Wing Fighter Airplane," NASA TN D-5409, Sept. 1969

${ }^{5}$ Adams, W. M., "Analytic Prediction of Airplane Equilibrium Spin Characteristics," NASA TN D-6926, 1972.

${ }^{6}$ Tischler, M. B., and Barlow, J. B., "Determination of Spin and Recovery Characteristics of a General Aviation Design," Journal of Aircraft, Vol. 18, No. 4, 1981, pp. 238-244

${ }^{7}$ Carroll, J. V., and Mehra, R. K., "Bifurcation Analysis of Nonlinear Aircraft Dynamics," Journal Guidance, Vol. 5, No. 5, 1982 , pp. $529-536$

${ }^{8}$ Guicheteau, P., "Bifurcation Theory Applied to the Study of Control Losses on Combat Aircraft," La Recherche Aerospatiale, Vol. 2, 1982, pp. 61-73.

${ }^{9}$ Guicheteau, P., "Bifurcation Theory in Flight Dynamics an Application to a Real Combat Aircraft," International Council of the Aeronautical Sciences, Paper 90-116, Sept. 1990.

${ }^{10}$ Planeaux, J. B., and Barth, T. J., "High Angle of Attack Dynamic Behavior of a Model High Performance Fighter Aircraft," AIAA Paper 88-4368, Aug. 1988.

"Planeaux, J. B., Beck, J. A., and Baumann, D. D., "Bifurcation Analysis of a Model Fighter Aircraft with Control Augmentation," AIAA Paper 90-2836, Aug. 1990.

${ }^{12}$ Guckenheimer, J., and Holmes, P., Nonlinear Oscillators, Dynamical Systems, and Bifurcations of Vector Fields, Springer-Verlag, 